both the martian atmosphere and hydrosphere through the direct impact of the magnetized solar wind on the ionosphere.

There is a special aspect of the martian system that makes its plasma environment particularly challenging to understand. Whereas the main features of other planetary environments are described well by the equations of magnetohydrodynamics, in which the motion of all particles is ascribed to a fluid flow, the observed scale sizes at Mars are comparable to the ions' local radii of gyration in the magnetic field $(1,000 \mathrm{~km}$ for a solar-wind ion). Thus the solar wind's interaction with Mars is modified by effects due to such finite Larmor radii ${ }^{7}$. One clear example of such a microphysics effect is the width of the foot boundary associated with the bow shock ${ }^{2,8}$. As is evident in Fig. 2, the shock foot (a shock precursor one ion-gyroradius wide)

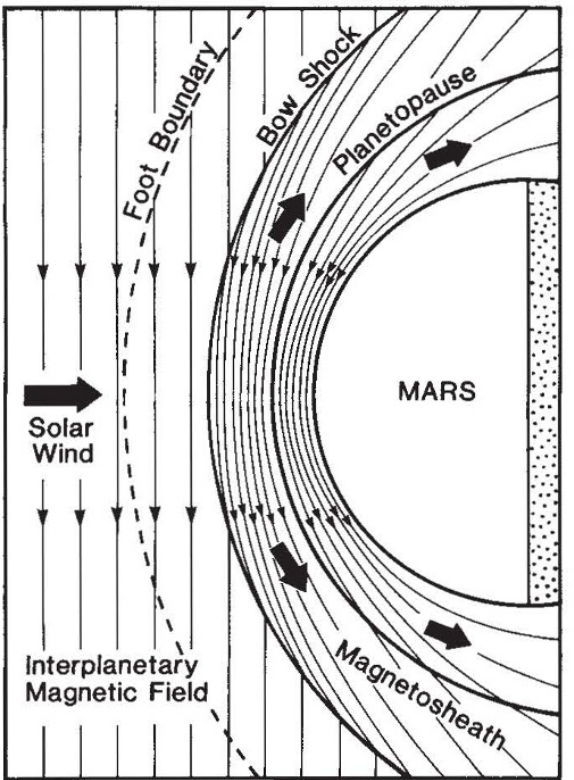

FIG. 2 The dominant dayside features of the solar-wind interactions with Mars seen by Phobos 2. The diagram emphasizes the main boundaries, the magnetic field geometry (arrowed lines) and the plasma flow (solid arrows).

is now comparable in width to the magnetosheath and is not an insignificant feature as it is at Earth.

Further important findings will follow these early analyses, but it is doubtful that Phobos 2 can answer the crucial question of whether Mars has a small or zero intrinsic magnetic field, because the $850 \mathrm{~km}$ periapsis (rather than the nominal 500 $\mathrm{km}$ ) was too high to probe the ionospheric regions where an internal field would be most detectable. Indeed a periapsis as low as $100-200 \mathrm{~km}$ may be required for a definitive answer, and this will have to await the planned NASA Mars Observer and Soviet MARS 94 missions.

Mark Saunders is in the Blackett Laboratory, Imperial College of Science, Technology and Medicine, London SW7 2BZ, UK.

\section{and martian surfaces}

\section{William B. McKinnon}

The loss of Phobos 2 in Mars orbit is a tragedy, of course, but it is not the end of the story. Although it is not widely recognized, many of the experiments on board were intended for Mars as well as Phobos, and it is probably these experiments that yielded the most new data and that will, eventually, provide the most new scientific understanding. The thermoscan (thermal infrared) images, in particular, are spectacular'. They instantly show much of the information previously painstakingly obtained by the Viking IRTM (infrared thermal mapper), and at better spatial resolution. And they allow one quickly to note the relatively warm and cool areas, interpret them in terms of albedos and thermal inertias (that is, soillike or rock-like thermal properties), and correlate all this with local geology.

The long history of Viking IRTM analyses shows that many factors, such as surface pressure, must be taken into account for proper interpretation, so it is premature to expect detailed geological interpretation at this time. Nevertheless, analysis of thermoscan images can draw on the IRTM experience. For example, high-resolution but broadband thermoscan radiometer data can be combined with lower resolution but multispectral IRTM data for maximum effect.

Phobos 2 also carried an infrared imaging spectrometer (ISM), and was the first spacecraft to do so at another planet. Several hundred spectra of Phobos were obtained along with some tens of thousands of spectra of Mars. The martian spectra sampled most of the principal geological terrains on the planet, with the unfortunate exception of the polar caps. In principle, it should be possible to use the details of the spectra collected to identify or place constraints on the types or amounts of various minerals in the martian soil and bedrock (if exposed). The example in the paper on the ISM results ${ }^{10}$ is the relative depth of the water hydration band near $3 \mu \mathrm{m}$. The authors are careful not to overinterpret the results, as the depth of an absorption feature does not uniquely indicate the relative amount of the absorber: grain size, the presence of opaque (and hence masking) minerals, degree of crystallinity and even temperature affect spectral band depths.

Refined analyses exploiting the results of years of ground-based spectroscopy

\footnotetext{
1. Sagdeev, R.Z. \& Zakharov, A.V. Nature 341, 581-585 (1989).

. Riedler, W. et al. Nature 341, 604-607 (1989)

3. Lundin, R. et al. Nature 341, 609-612 (1989).

3. Lundin, R. et al. Nature 341, 609-612 (1989).

5. Alfven, H. Tellus 9, 92-96 (1957)

6. Luhmann, J.G. et al. J. geophys. Res. 92, 8545 (1987)

7. Moses, S.L. et al. Geophys. Res. Lett. 15, 429 (1988)
}

8. Grard, R. et al. Nature 341, 607-609 (1989) should provide more secure identification of primary igneous minerals, and most importantly, perhaps shed some light on the controversy surrounding identification of carbonate-bearing minerals in the martian soil. Carbonate is, naturally enough, a sink for atmospheric $\mathrm{CO}_{2}$, and its presence may indicate a more aqueous (and clement) Mars in the past.

The spacecraft brought a multichannel infrared radiometer to Mars as well, but the results presented so far concern only Phobos $^{11}$. The data indicate intriguing inhomogeneities in the inner moon's structure and composition. ISM data for Phobos also indicate variations in hydration and silicate spectral features ${ }^{10}$. The 3$\mu \mathrm{m}$ hydration absorption feature is markedly weaker than for Mars, which may at first seem surprising given that Phobos (and Deimos) are often viewed as captured carbonaceous asteroids and that carbonaceous meteorites are mostly water-rich clay minerals. However, the surfaces of many carbonaceous asteroids, particularly those in the outer asteroid belt, are rather dry, and ground-based spectroscopy has recently shown that Deimos possesses at most only a small absorption feature at 3- $\mu \mathrm{m}$, implying that it is relatively dry ${ }^{12}$. The ground-based experiment is too difficult to do for Phobos because of scattered light from Mars, but the telescopic and ISM data combined may indicate that Phobos and Deimos are similar carbonaceous bodies with closer to anhydrous surfaces.

It is also interesting to note that the Phobos mission indicates that the moon's density is about $1.95 \mathrm{~g} \mathrm{~cm}^{-3}$, which is somewhat less than that determined by Viking. The imaging team reports that this is consistent with substantial internal porosity for a rock body ${ }^{13}$, which is true, but some noted astronomers still favour an icy interior. Paradoxically, an icy interior would not conflict with a dry surface, because this is what is expected of outer belt asteroids according to current thinking ${ }^{14}$. The prospect of icy moons circling a thirstless desert below is only one of many martian mysteries to be explored by further analysis of Phobos 2 data.

William B. McKinnon is in the Department of Earth and Planetary Sciences and McDonnell Center for Space Sciences, Washington University, Saint Louis, Missouri 63130, USA.

9. Selivanov, A.S. et al. Nature 341, 593-595 (1989). 10. Bibring, J.-P. et al. Nature 341, 591-593 (1989).

11. Ksanfomality, L.V. et al. Nature 341, 588-591 (1989).

12. Bell, J.F., Piscitelli, J.R. \& Lebofsky, L.A. Lunar planet Sci. XX, 58-59 (1989)

13. Avanesov, G.A. et al. Nature 341, 585-587 (1989).

14. Lebofsky, L.A. et al. in Origin and Evolution of Planet and Satellite Atmospheres (eds Atreya, S. K. et al.) 192-229 (University of Arizona Press, Tucson, 1989). 